\title{
Kinetic pathways of the Nematic-Isotropic phase transition as studied by confocal microscopy on rod-like viruses.
}

\author{
M. Paul Lettinga†, Kyongok Kang $\dagger$, Arnout Imhof $\ddagger$, Didi \\ Derksłand Jan K. G. Dhont $\dagger$ \\ †IFF, Institut Weiche Materie, Forschungszentrum Jülich,D-52425 Jülich, \\ Germany \\ ‡Soft Condensed Matter, Debye Institute, Utrecht University, Princetonplein 5, \\ 3584 CC Utrecht, The Netherlands \\ E-mail: p.lettinga@fz-juelich.de
}




\begin{abstract}
We investigate the kinetics of phase separation for a mixture of rodlike viruses $(f d)$ and polymer (dextran), which effectively constitutes a system of attractive rods. This dispersion is quenched from a flow-induced fully nematic state into the region where the nematic and the isotropic phase coexist. We show experimental evidence that the kinetic pathway depends on the overall concentration. When the quench is made at high concentrations, the system is meta-stable and we observe typical nucleation-and-growth. For quenches at low concentration the system is unstable and the system undergoes a spinodal decomposition. At intermediate concentrations we see the transition between both demixing processes, where we locate the spinodal point.
\end{abstract}

PACS numbers: 82.60.Nh,82.70.Dd,64.70.Md

\title{
1. Introduction
}

Systems that are quenched into a state where at least one order parameter is unstable undergo spinodal phase separation. Here, the initially homogeneous system is unstable against fluctuations of arbitrary small amplitude, and phase separation sets in immediately after a quench. In the initial stage of phase separation an interconnected "labyrinth structure" of regions with somewhat higher and lower values of the order parameter is observed. For quenches where the system becomes meta-stable, phase separation is initiated by fluctuations with a sufficiently large amplitude. Since such fluctuations have a small probability to occur, phase separation sets in after a certain delay time, referred to as the induction time. Here, nuclei are formed throughout the volume which grow when they are sufficiently large. The two different mechanisms of phase separation (spinodal decomposition and nucleation-and-growth) can thus be distinguished during the initial stages of phase separation from (i) the difference in morphology (interconnected structures versus growth of isolated nuclei) and (ii) the delay time before phase separation sets in (no delay time for spinodal decomposition and a finite induction time for nucleation-and-growth). As Onsager showed in 1949 [1, the situation is different when the particles are not spherical in shape, i.e. disk-like or elongated particles. Here the system can become unstable or meta-stable with respect to fluctuations in orientation. These orientational fluctuations drive concentrations differences, resulting in a phase with high concentration and orientational order, the nematic phase, and a phase with low concentration and no orientational order, the isotropic phase. For very long and thin rods with short-ranged repulsive interactions, the binodal concentrations, i.e. the concentrations of the isotropic and nematic phases in equilibrium after phase separation is completed, have been determined using different approximations in minimizing Onsager's functional for the free energy (see ref. [2] and references therein), while for shorter rods computer simulations have been performed to obtain binodal concentrations [3, 4]. Also the spinodal concentration where the isotropic phase becomes unstable has been found [1, 5].

Binodal points are relatively easy to determine experimentally, since they are given by the concentrations of the bottom and top phase after phase separation. In contrast, it is not at all straightforward to obtain spinodal points, since one would ideally $\mathrm{n}$ like to perform a concentration quench from low or high concentration into the two-phase region, where the initial state is isotropic or nematic, respectively. In a recent paper such a kind of 'quench' was performed by inducing polymerization of short actin chains [6], and tactoids and spinodal structures were observed. Signatures 


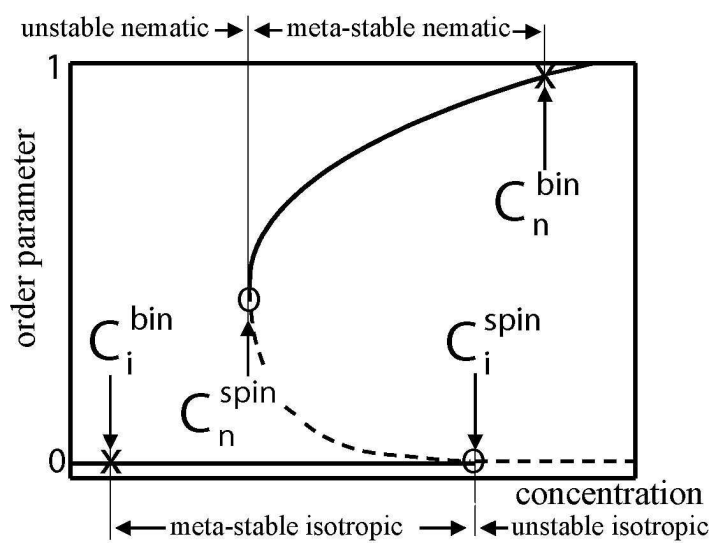

Figure 1. The bifurcation diagram, where the orientational order parameter $P_{2}$ is plotted against concentration. Indicated are the various meta- or unstable regions for the two different initial states of the homogeneous suspension. The points marked by $\mathrm{X}$ and $\mathrm{O}$ are spinodal and binodal points, respectively.

of spinodal decomposition have also been obtained for boehmite rods, by homogenizing a phase separated system and sequential polarization microscopy and Small Angle Light Scattering measurements [7. Alternatively, external fields like shear flow $[8]$ and a magnetic field [9] 10 can be applied to prevent a system from phase separation and to stabilize the nematic phase. After cessation of such an external field the nematic phase will become unstable or meta-stable, depending on the constitution of the sample, and phase separation sets in. In this paper we induce a fully nematic phase with a well defined director by imposing shear flow to a dispersion of colloidal rods. We use $f d$-viruses as system, since the equilibrium phase behavior concerning the binodal points, has been well understood on the basis of Onsager theory [11, 12]. Polymer is added to the dispersion in order to widen the region of isotropic-nematic phase coexistence, which facilitates the phase separation experiments 13. We perform quenches of a flow aligned initial state to zero shear, which renders the system unstable or meta-stable to fluctuations in the orientation, depending on the concentration of rods. As a consequence phase separation sets in, which we observe by Confocal Laser Scanning Microscopy (CSLM). We perform this experiment for different concentrations, throughout the region of phase coexistence. Our results illustrate the difference between nucleation-and-growth and spinodal decomposition in the case of demixing elongated particles, and result in the determination of the nematic-isotropic spinodal point.

\section{On the Instability of Initial States}

A convenient way to analyze the stability of a homogeneous initial state is to derive an equation of motion for the order parameter tensor,

$$
\mathbf{S}_{0}(t)=\oint d \hat{\mathbf{u}} \hat{\mathbf{u}} \hat{\mathbf{u}} P_{0}(\hat{\mathbf{u}}, t) .
$$

The largest eigenvalue $P_{2}$ of the tensor $\mathbf{Q}_{0}(t)=\frac{3}{2}\left[\mathbf{S}_{0}(t)-\frac{1}{3} \hat{\mathbf{I}}\right]$ (where $\hat{\mathbf{I}}$ is the identity) measures the degree of alignment. For the isotropic state $P_{2}=0$, while for a perfectly 
aligned state $P_{2}=1$. A stability analysis of stationary solutions of this equation of motion is most conveniently made on the basis of a bifurcation diagram [5], where $P_{2}$ for stationary solutions is plotted against the concentration. A schematic bifurcation diagram is given in fig 1. The two solid lines represent stable stationary solutions of the equation of motion, while the dotted lines represent unstable stationary solutions. The isotropic state ceases to be stable above the concentration indicated as $C_{i}^{\text {spin }}$, while the nematic state becomes unstable at concentrations lower than $C_{n}^{\text {spin }}$. Above $C_{i}^{\text {spin }}$, the isotropic state is still a stationary solution, but is now unstable. Below $C_{n}^{\text {spin }}$, on the contrary, there is no unstable nematic state that is a stationary solution of the equation of motion. The two spinodal concentrations $C_{i}^{\text {spin }}$ and $C_{n}^{\text {spin }}$ are connected by a separatrix which separates the basins of attraction for the isotropic and nematic state. A homogeneous initial state above this separatrix develops a higher degree of alignment, while an initial state below the separatrix becomes more isotropic.

Note that the bifurcation diagram relates to homogeneous systems. In an experiment, starting from a homogeneous state, inhomogeneities develop simultaneously with the change of the order parameter of the otherwise homogeneous system. In equilibrium, after completion of phase separation, there is an isotropic phase with concentration $C_{i}^{b i n}$ in coexistence with a nematic phase with concentration $C_{n}^{b i n}$. One can either start from a stationary state, in which case $P_{0}$ in eq. (11) is independent of time, or from a non-stationary state, like a nematic state with a concentration lower than $C_{n}$, in which case the time dependence of the temporal evolution of alignment of the otherwise homogeneous system couples to the evolution of inhomogeneities through the time dependence of $P_{0}$.

In this paper we prepare an initial nematic state shearing a suspension at large enough shear rate such that the induced nematic phase is stable against phase separation (see refs. 14 for a discussion of the bifurcation diagram for sheared systems), and then quench to zero shear rate. For this initial state it is expected that spinodal decomposition occurs at lower concentrations, while nucleation and growth is observed at higher concentrations. For an isotropic initial state this would be reversed : spinodal decomposition at high concentrations and nucleation and growth at lower concentrations. The observed phase separation kinetics thus depends crucially on the preparation of the initial state of the suspension.

\section{Materials and methods}

As model colloidal rods we use $f d$-virus particles which were grown as described elsewhere 13. A homogeneous solution of $22.0 \mathrm{mg} / \mathrm{mL} \mathrm{fd}$-virus and $12.1 \mathrm{mg} / \mathrm{mL}$ of Dextran (507 kd, Sigma-Aldrich) in $20 \mathrm{mM}$ tris buffer at $\mathrm{pH} 8.15$ with $100 \mathrm{mM}$ $\mathrm{NaCl}$ is allowed to macroscopically phase separate. This concentration of $f d$-virus is exactly in the biphasic region, which is very small when no polymer is added, namely between 21 and $23 \mathrm{mg} / \mathrm{mL}$. Due to the added polymer, the binodal points shift to 17 and $30 \mathrm{mg} / \mathrm{mL}$, respectively. New dispersions are prepared by mixing a known volume of the coexisting isotropic and nematic bulk phases. The relative volume of nematic phase in this new dispersion is denoted as $\varphi_{\text {nem }}$.

For the microscopic observations we used a home-built counter rotating cone-plate shear cell, placed on top of a Leica TCS-SP2 inverted confocal microscope. This cell has a plane of zero velocity in which objects remain stationary with respect to the microscope while shearing. For details of the setup we refer to reference 15. For the measurements described here we used confocal reflection mode at a wavelength of 488 
Table 1. Overview of the used samples.

\begin{tabular}{|c|c|c|c|c|c|}
\hline Code & $\varphi_{\text {nem }}^{5}$ & $\varphi_{\text {nem }}^{4}$ & $\varphi_{\text {nem }}^{3}$ & $\varphi_{\text {nem }}^{2}$ & $\varphi_{\text {nem }}^{1}$ \\
\hline$f d(\mathrm{mg} / \mathrm{mL})$ & 29.5 & 28.1 & 25.8 & 23.6 & 19.3 \\
\hline$\varphi_{\text {nem }}$ & 0.96 & 0.85 & 0.68 & 0.52 & 0.18 \\
\hline
\end{tabular}

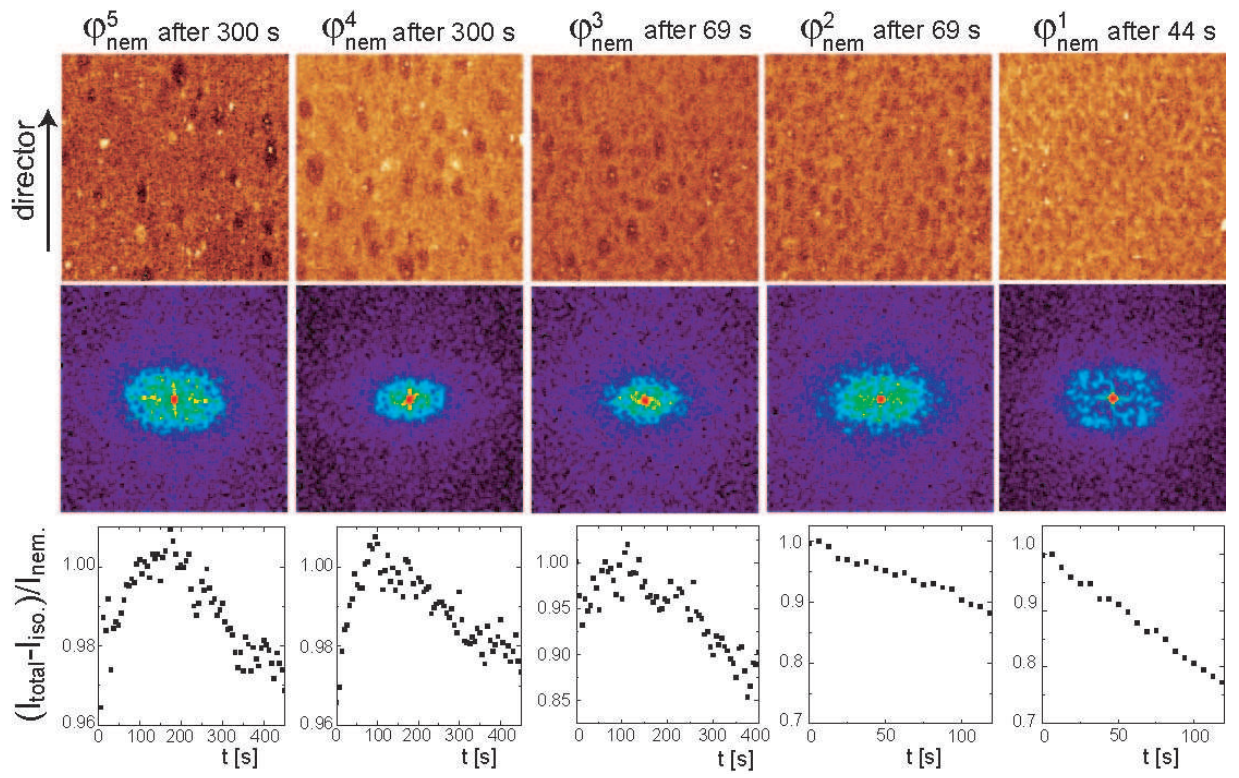

Figure 2. The initial stages of phase separation for five different concentrations after a quench from a flow aligned nematic phase to zero shear. The top row shows the micrographs taken by reflection confocal scanning laser microscopy (field of view $=110 \mu \mathrm{m}$ ); the middle row shows the fourier transform of the micrographs; the bottom row plots the mean intensity of the micrographs minus the mean intensity for the isotropic phase, normalized by the initial intensity of the nematic phase.

$\mathrm{nm}$. Quench experiments were done as follows. Samples were first sheared at a high rate of $10 \mathrm{~s}^{-1}$ for several minutes. The shear was then suddenly stopped, after which images were recorded at regular time intervals. These images were parallel to the flow-vorticity plane. The table gives an overview of the concentrations where quench experiments have been performed.

\section{Results}

In the top row of Figure 2 we show micrographs of the initial stage of phase separation for five different concentrations taken after a shear rate quench from a high shear rate, where the nematic state is stable for each concentration, to zero shear. These images show the flow (vertical) - vorticity (horizontal) plane at a given time after the quench. Thus the director of the initial nematic phase lies in the vertical direction. Fourier transforms of the images are plotted in the second row of Fig. 22 The background is corrected for by subtracting the Fourier transform of the first frame. The third row 


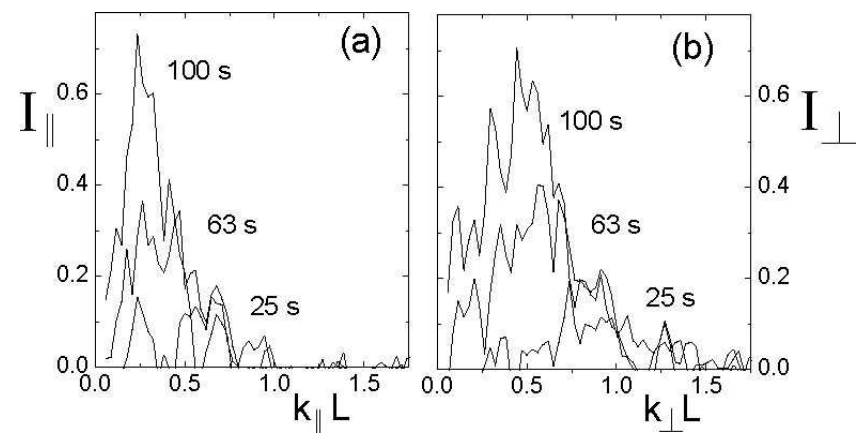

Figure 3. The cross section of the Fourier transform parallel (a) and perpendicular (b) to the director for $\varphi_{\text {nem }}^{1}$. The length is scaled by the rod length $L$.

plots the development of the total intensity of the images minus the intensity in the isotropic phase, as determined from an isolated isotropic region, normalized by the initial nematic intensity. Qualitatively the difference between the concentrations is obvious. In the first two images, i.e. the two highest concentrations, isolated dark ellipsoidal structures can be seen on a bright back ground. These are droplets of the isotropic phase referred to as tactoids. The number of tactoids increases when the concentration is decreased (b and c) until the structures become interconnected ( $\mathrm{d}$ and e). This also follows from the fourier transform of the pictures where a ring is detected for the lowest concentration and a constant increasing intensity towards $K=0$ for the highest concentration. The time scale at which the inhomogeneities are formed also changes. As can be seen in the third row of Fig. 2 the high concentrations all show an induction time before the phase separation sets in, while for the low concentrations phase separation sets in immediately. Note also the times at which the images in Fig. 2 were taken. The isolated nuclei and the induction time are typical for nucleationand-growth, while the interconnected structures and immediate phase separation are typical for spinodal decomposition.

We use the Fourier transform of the images as shown in Fiq. 2 to quantify the phase separation processes. The interesting quantity for nucleation-and-growth is the width of the Fourier transform, $\Delta k$, which is a measure for the anisotropic form factor of the nuclei. Alternatively one could determine the average size of the features in real space, but due to the low contrast this is difficult. For spinodal decomposition the interesting quantity is the wavevector at which the fourier transform reaches its maximum, $k_{\max }$, quantifying the fastest growing concentration fluctuation. In both cases the fit of the Fourier transform should be performed in two dimensions, since the initial state is anisotropic. Therefore we took cross sections parallel and perpendicular to the director in the Fourier domain, i.e. the vertical and horizontal in Fig. 2] middle row, to determine $k_{\max }$. Typical cross sections for are shown in Fig. 3 where the wavevector $k$ is scaled by the rod length $L$. To determine $\Delta k$, we performed a 2-D gaussian fit around the origin of the Fourier transforms. Results of a 2-D gaussian fit of the Fourier transform around the origin are shown for the higher concentrations in Fig. 桄 and b, plotting the width in the direction of the director and perpendicular to the director, respectively. $k_{\max }$ as found from fits of the cross sections parallel

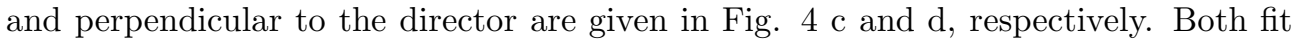



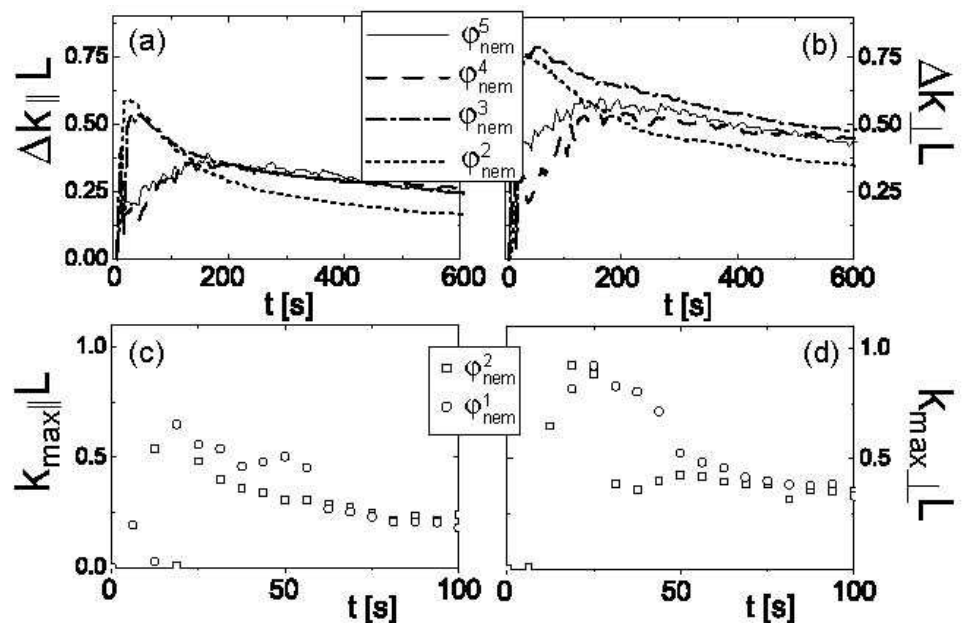

Figure 4. Width of the 2-D gaussian fit, $\Delta k L$, of the Fourier transform parallel (a) and perpendicular (b) to the director for the higher concentrations for the higher concentrations. Wavevector $k_{\max } L$ where the intensity is maximum for the cross sections parallel (c) and perpendicular (b) to the director for the lower concentrations.
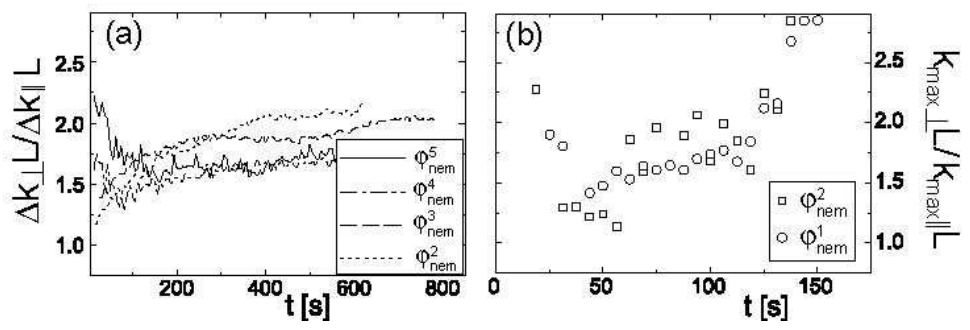

Figure 5. (a) The ratio $\Delta k_{\perp} L / \Delta k_{\|} L$ for the higher concentrations and (b) the ratio $k_{\max , \perp} L / k_{\max , \|} L$ for the lower concentrations.

procedures result in an anisotropic morphology as can be seen in Fig. 5 where we plotted $\Delta k_{\perp} L / \Delta k_{\|} L$ and $k_{\max , \perp} L / k_{\max , \|} L$.

The late stages of the different phase separation processes also show some interesting phenomenology, as can be seen in Fig. 6. For spinodal decomposition we observe first a growing of the interconnected structures, which then break down into tactoids. Later on tactoids coalesce, and they become more spherical with increasing size. Note that these tactoids contain the nematic phase and not the isotropic phase, as observed for the nucleation-and-growth process at higher concentrations. In the late stage of nucleation-and- growth, i.e. at high concentrations, we see that coalescence of tactoids containing the isotropic phase as shown in the bottom row of Fig. [6] is favorable when two tactoids meet somewhat from the middle. In this case the rod orientation near both features is similar and the barrier which has to be overcome for coalescence is low. 

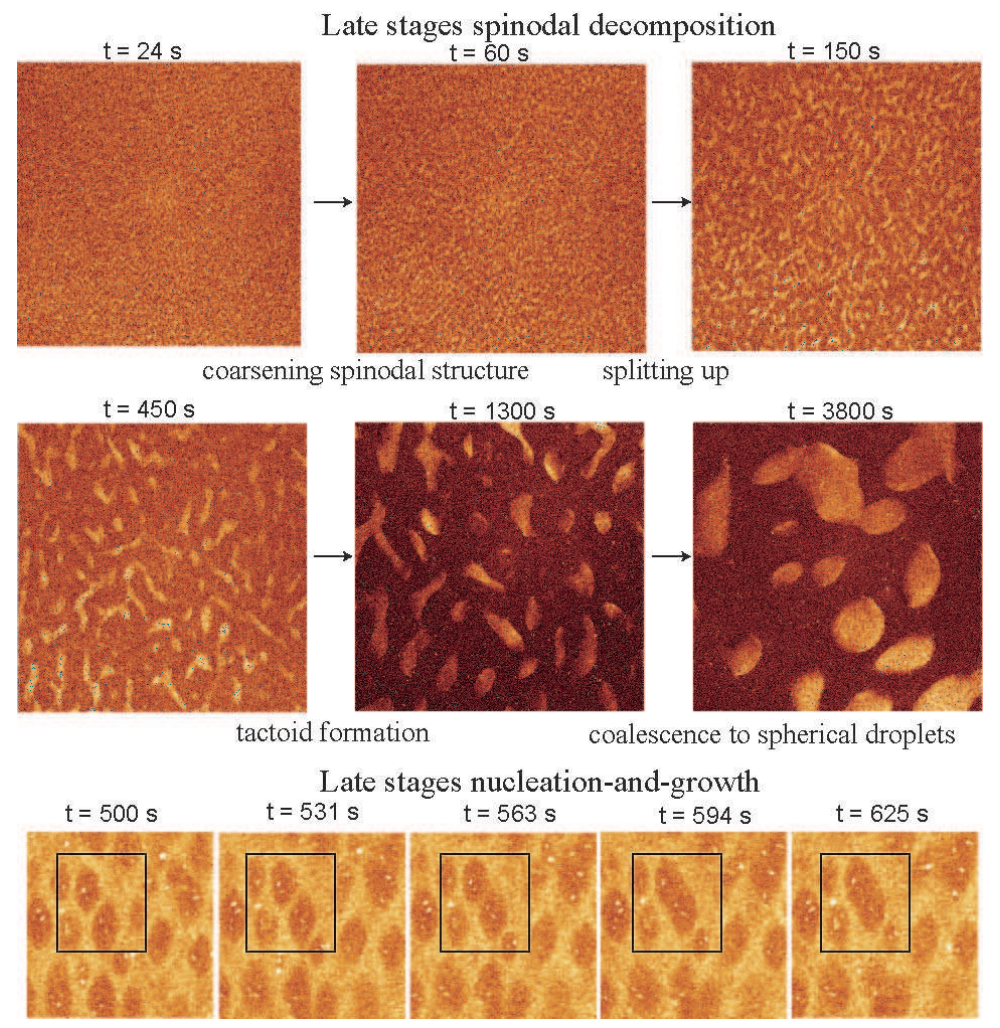

Figure 6. The late stages for spinodal decomposition in the top two rows $\left(\varphi_{\text {nem }}^{1}\right.$, field of view $=375 \mu \mathrm{m})$, and coalescence of tactoids in the bottom row $\left(\varphi_{\text {nem }}^{5}\right.$, field of view $=73 \mu \mathrm{m})$.

\section{Discussion}

On the basis of these observations we can now locate the metastable region, i.e. where the system has to overcome a free energy barrier, and the unstable region, where there is no such barrier. At the high concentrations $\left(\varphi_{n e m}^{5}, \varphi_{n e m}^{4}\right)$ the system is meta-stable, which is reflected by the observed isolated structures formed (top row in Fig 22) and the induction time (bottom row in Fig 2). With decreasing concentration the system approaches the unstable region: the number of nuclei increases while the induction time decreases and finally vanishes. The lowest concentration $\varphi_{n e m}^{1}$ is clearly unstable after cessation of the flow. It shows all the features typical for spinodal decomposition: phase separation immediately sets in throughout the whole sample, with a typical length scale which is characterized by the scattering ring observed in the Fourier transform. It can be shown, in fact, that the observed phase separation process for the lowest concentration has features typical for the spinodal decomposition of rods, as derived recently from a microscopic theory by one of the authors 16. This will be the subject of a following paper 17.

In the intermediate region it is difficult to judge from the morphology if nucleation-and-growth takes place or spinodal decomposition, since it is difficult to distinguish between a high number of tactoids and an interconnected structure. 
However, $\varphi_{\text {nem }}^{3}$ shows a short induction time after the quench after which clearly separated tactoids are formed, while for $\varphi_{\text {nem }}^{2}$ phase separation immediately sets in showing ellipsoidal structures which clearly 'influence' each other. Moreover, Figures [4 shows that the size of the structures formed in $\varphi_{\text {nem }}^{3}$ coincides after some time with the clearly nucleated structures of $\varphi_{\text {nem }}^{4}$ and $\varphi_{\text {nem }}^{5}$, while the size of the structures formed in $\varphi_{\text {nem }}^{2}$ coincides with samples which clearly show spinodal decomposition. Thus, we locate the transition from meta-stable to unstable, i.e. the spinodal point, between at $23.5 \mathrm{mg} / \mathrm{mL}$ and $25.8 \mathrm{mg} / \mathrm{mL}$. This is the first experimental observation of the spinodal point in a rod-like system. We should mention at this point that in fact our sample consists of a mixture of rods and polymer. Addition of the polymer causes a widening of the biphasic region [13, i.e. a shift of the binodal points. It is now interesting to see that the high concentration binodal shifts as much as from $23 \mathrm{mg} / \mathrm{mL}$ to $30 \mathrm{mg} / \mathrm{mL}$. In contrast, the high concentration binodal point, $C_{n}^{b i n}$, shifts from a concentration between $21 \mathrm{mg} / \mathrm{mL}$ and $23 \mathrm{mg} / \mathrm{mL}$ to somewhere between $23.5 \mathrm{mg} / \mathrm{mL}$ and $25.8 \mathrm{mg} / \mathrm{mL}$. This leads to the interesting conclusion that the shift of the high concentration binodal point, $C_{n}^{b i n}$, due to the attraction between the rods, is considerable compared to the shift of the high concentration spinodal point, $C_{n}^{\text {spin }}$. In other words, making the rods attractive causes a widening of the meta-stable region, while the unstable region remains unaffected. Addition of more polymer will result in more complex kinetics as described in reference [18.

Interestingly, for all concentrations we observe that the morphology of the phase separating system is anisotropic. This is most clear for the highest concentrations, where the tactoids all point upwards, i.e. in the direction of the director of the surrounding nematic phase. Also the Fourier transforms for the lower concentrations show deformed intensity rings in fourier space (most right FFT image in Fig. 2). Moreover, also the kinetics of the phase separation is fastest in the direction of the nematic director. This follows for instance from the ratio of $\mathbf{k}_{\max }$ as plotted in $5 \mathbf{b}$, which increases in time. In other words, for all concentrations phase separation is anisotropic, due to residual alignment after the quench of the initially strongly sheared suspension, and not isotropic as is the case for spheres [19.

The length of the first observed tactoids just below $C_{n}^{\text {bin }}$ is about 12 times the rod length, while just above $C_{n}^{s p i n}$ it is seven times the rod length. The thickness is about two third of the length in both cases. Typical length scales for the initial spinodal morphology are not more than six rod lengths. These sizes seem to be quite small, considering also the random orientation of the rods in the isotropic phase, but it is in accordance with a the microscopic theory for spinodal decomposition of rods [16. It does suggest that we really image the initial stage. The breaking up of the spinodal structure into nematic tactoids and the sequential growth in the late stage of spinodal decomposition seems surprising since for dispersions of spheres only coalescence and macroscopic phase separation would be observed. However, a similar order of events has been observed for polymer mixtures with thermotropic liquid crystals [20]. Simulations on such mixtures show that the break down is due to the effect of the flow-alignment coupling, and not primarily due to elastic effects 21]. An explanation in the same line was given by Fukuda in a numerical treatment of timedependent Ginzburg-Landau equations of liquid crystalline polymers [22]. The volume dependence of the morphology in the final stage can be explained by the competition between the interfacial tension and nematic elasticity of the tactoids [23. 
Kinetic path way of the Nematic-Isotropic phase transition

\section{Conclusion}

We studied the kinetics of the nematic-isotropic phase transition of a dispersion of $f d$ virus particles with added polymer after shear quenches into the two-phase region. By varying the equilibrium rod concentration $\varphi_{n e m}$ we were able to detect a nucleationand-growth mechanism for high $\varphi_{\text {nem }}$, spinodal decomposition for low $\varphi_{n e m}$, and the transition between the two processes. In this way we were able to trace for the first time the nematic-isotropic spinodal point $C_{n}^{s p i n}$. Thus, we found that addition of polymer widens the meta-stable region greatly. Furthermore, we showed that the phase separation is strongly influenced by the director of the initial nematic state. The nematic phase also influences the late stages of spinodal decomposition, causing a splitting up of the interconnected structures.

\section{Acknowledgments}

This work was performed within the framework of the Transregio SFB TR6, "Physics of colloidal dispersions in external fields".

[1] L. Onsager. The effect of shape on the interaction of colloidal particles. Annals of the New York academy of science, 51:62-659, 1949.

[2] G. J. Vroege and H. N. W. Lekkerkerker. Phase transitions in lyotropic colloidal and polymer liquid crystals. Rep. Prog. Phys., 55:1241-1309, 1992.

[3] P. Bolhuis and D. Frenkel. Tracing the phase boundaries of hard spherocylinders. J. Chem. Phys., 106(2):666-687, 1997.

[4] H. Graf and H. Löwen. Phase diagram of tobacco mosaic virus solutions. Phys. Rev. E, 59(2):1932-1942, 1999.

[5] R. F. Kayser Jr. and H. J. Raveché. Bifurcation in onsager's model of the isotropic-nematic transition. Phys. Rev. A, 17:2067-2072, 1978.

[6] J. Viamontes and J. X. Tang. Formation of nematic liquid crystalline phase of f-actin varies from continuous to biphasic transition. http://arxiv.org/abs/cond-mat/0506813 2005.

[7] M. P. B. van Bruggen, J. K. G. Dhont, and H. N. W. Lekkerkerker. Morphology and kinetics of the isotropic-nematic phase transition in dispersions of hard rods. Macromolecules, 32:22562264, 1999.

[8] T. A. J. Lenstra, Z. Dogic, and J. K. G. Dhont. Shear-induced displacement of isotropic-nematic spinodals. J. Chem. Phys., 114(22):10151-10162, 2001.

[9] J. Tang and S. Fraden. Magnetic-field-induced phase transition in a colloidal suspension. Phys. Rev. Lett., 71(21):3509-3512, 1993.

[10] B. J. Lemaire, P. Davidson, J. Ferré, J.-P. Jamet, D. Petermann, P. Panine, I. Dozov, D. Stoenescuc, and J.-P. Jolivet. The complex phase behaviour of suspensions of goethite ( $\alpha$-feooh) nanorods in a magnetic field. Faraday Discuss., , ,, 128:271-283, 2005.

[11] S. Fraden. Observation, Prediction, and Simulation of Phase Transitions in Complex Fluids, volume 460 of NATO-ASI - Series C, pages 113-164. Kluwer Academic Publishers, Dordrecht, $\mathrm{m}$. baus and l. f. rull and j. p. ryckaert, edition, 1995,.

[12] Z. Y. Chen. Nematic ordering in semiflexible polymer chains. Macromolecules, 26:3419-3423, 1993.

[13] Z. Dogic, K. R. Purdy, E. Grelet, M. Adams, and S. Fraden. Isotropic-nematic phase transition in suspensions of filamentous virus and dextran. Phys. Rev. E, 69:051702, 2004.

[14] J. K. G. Dhont and W. J. Briels. Viscoelasticity of suspensions of long, rigid rods. Colloid Surface A, 213(2-3):131-156, 2003.

[15] D. Derks, H. Wisman, A. van Blaaderen, and A. Imhof. Confocal microscopy of colloidal dispersions in shear flow using a counter-rotating coneplate shear cell. J. Phys.: Condens. Matter, 16:S3917-S3927, 2004.

[16] J. K. G. Dhont and W. J. Briels. Isotropic-nematic spinodal decomposition kinetics. accepted in Phys. Rev. E.

[17] M.P. Lettinga, K. Kang, P. Holqvist, A. Imhof, D. Derks, and J.K.G. Dhont. Nematic-isotropic spinodal decomposition of rod-like viruses. submitted.

[18] Z. Dogic. Surface freezing and a two-step pathway of the isotropic-smectic phase transition in colloidal rods. Phys. Rev. Lett., 91(16):165701, 2003. 
[19] D. G. A. L. Aarts and H. N. W. Lekkerkerker. Confocal scanning laser microscopy on fluid-fluid demixing colloid-polymer mixtures. J. Phys.: Condens. Matter, 16:S4231, 2004.

[20] A. Nakai, T. Shiwaku, W. Wang, H. Hasegawa, and T. Hashimoto. Process and mechanism of phase separation in polymer mixtures with a thermotropic liquid crystalline copolyester as one component. Macromolecules, 29:5990-6001, 1996.

[21] T. Araki and H. Tanaka. Nematohydrodynamic effects on the phase separation of a symmetric mixture of an isotropic liquid and a liquid crystal. Phys. Rev. Lett., 93(1):015702, 2004.

[22] J. Fukuda. Phase separation kinetics of liquid crystalline polymers: Effect of orientational order. Phys. Rev. E, 59(3):3275-3288, 1999.

[23] P. Prinsen and P. van der Schoot. Shape and director-field transformation of tactoids. Phys. Rev. E, 68:021701, 2003. 